\title{
Measurement of the top-quark pair production cross section in ATLAS
}

\section{M.J.Costa*}

Instituto de Fisica Corpuscular, University of Valencia and CSIC, Valencia, Spain

E-mail: Maria.Jose.Costa@ific.uv.es

Measurements of the top-quark pair-production in proton-proton collisions at $\sqrt{s}=7 \mathrm{TeV}$ with the ATLAS detector at the Large Hadron Collider are presented. The cross-section is measured in several channels, including the single lepton and dilepton channel, some using information from $b$-tagging. A search for top-quark pair production in the all hadronic channel is also shown.

The 2011 Europhysics Conference on High Energy Physics-HEP 2011,

July 21-27, 2011

Grenoble, Rhône-Alpes France

\footnotetext{
*Speaker.

$\dagger$ On behalf of the ATLAS Collaboration.
} 


\section{Introduction}

The measurement of the top quark pair production cross-section is one of the key milestones for the Large Hadron Collider (LHC) physics programme. The precision achieved in the theoretical calculcations of the cross-section is now at the level below 10\%. Therefore, a precise measurement of the cross-section allows for precision tests of perturbative QCD. In addition, $t \bar{t}$ production is an important background to the search for Higgs boson and various searches for physics beyond the Standard Model (SM). New physics may also give rise to additional $t \bar{t}$ production mechanisms or modification of the top quark decay channels, which can affect the measured $t \bar{t}$ cross-section.

In the SM the $t \bar{t}$ production cross-section in $p p$ collisions is calculated to be $165_{-16}^{+11} \mathrm{pb}$ [1, 2, 3] at a centre-of-mass energy $\sqrt{s}=7 \mathrm{TeV}$ assuming a top quark mass of $172.5 \mathrm{GeV}$. Top quarks are predicted to decay to a $W$-boson and a $b$-quark $(t \rightarrow W b)$ nearly $100 \%$ of the time. Events with a $t \bar{t}$ pair can be classified as 'single lepton', 'dilepton', or 'all hadronic' according to the decays of the two $W$-bosons: each can decay into quark-antiquark pairs $\left(W \rightarrow q_{1} \bar{q}_{2}\right)$ (in about $2 / 3$ of the decays) or a lepton-neutrino pair ( $W \rightarrow \ell v$ ) (in about $1 / 3$ of the cases). Only the leptonic decays of the $W$ to an electron or muon are considered, including the small contribution from $W \rightarrow \tau \rightarrow e$ and $W \rightarrow \tau \rightarrow \mu$.

At the Tevatron the $t \bar{t}$ cross-sections at $\sqrt{s}=1.8 \mathrm{TeV}$ and at $\sqrt{s}=1.96 \mathrm{TeV}$ have been measured by CDF [ $₫$, 5] and and D [6, 7] in most channels. Recently, CMS and ATLAS presented the first measurements of the $t \bar{t}$ cross-section at $\sqrt{s}=7 \mathrm{TeV}$ at the LHC [8, 9].

This document summarizes the results obtained by ATLAS on the $t \bar{t}$ production cross-section available in July 2011. That includes the measurements obtained in the dilepton channel using 0.70 $\mathrm{fb}^{-1}$ of data collected in 2011 [10] (Section 2], those obtained in the single lepton channel with the full $35 \mathrm{pb}^{-1}$ of data recorded in 2010 [11, 12] (Section B), and a search for $t \bar{t}$ production in the all-hadronic channel using the 2010 dataset [13]. A combination of the measurements obtained in the di-lepton and single lepton channels [14] is also presented in Section $甘$

\section{Measurement of the $t \bar{t}$ production cross-section in the dilepton channel}

The $t \bar{t}$ production in the dilepton channel, which corresponds to about $5 \%$ of the $t \bar{t}$ decays, yields an event with two opposite-sign leptons, unbalanced transverse momentum indicating the presence of neutrinos from the $W$-boson decays and two $b$-quark jets. These final states can be selected with a good signal-to-background ratio using simple kinematic requirements on the final state objects. With the additional requirement of the presence of a jet consistent with arising from a $b$ quark, the signal-to-background ratio can be further improved. Two complementary measurements with and without b-tagging requirement suffering from different systematic uncertainties were performed, in which the leptons include both reconstructed electrons and muons, using a total of $0.70 \mathrm{fb}^{-1}$ of data collected in 2011 .

The analysis requires collision data selected by an inclusive single lepton trigger $(e$ or $\mu$ ) that is fully efficient for leptons satisfying $p_{T}>25 \mathrm{GeV}$. One of the well-identified leptons and the trigger object are required to match within $\Delta R<0.15$. The candidate events contain exactly two oppositely-sign leptons and at least two jets. In order to reject backgrounds from bottom quark production and vector meson decays, events are required to have $m_{\ell \ell}>15 \mathrm{GeV}$. Events with $e e$ and 

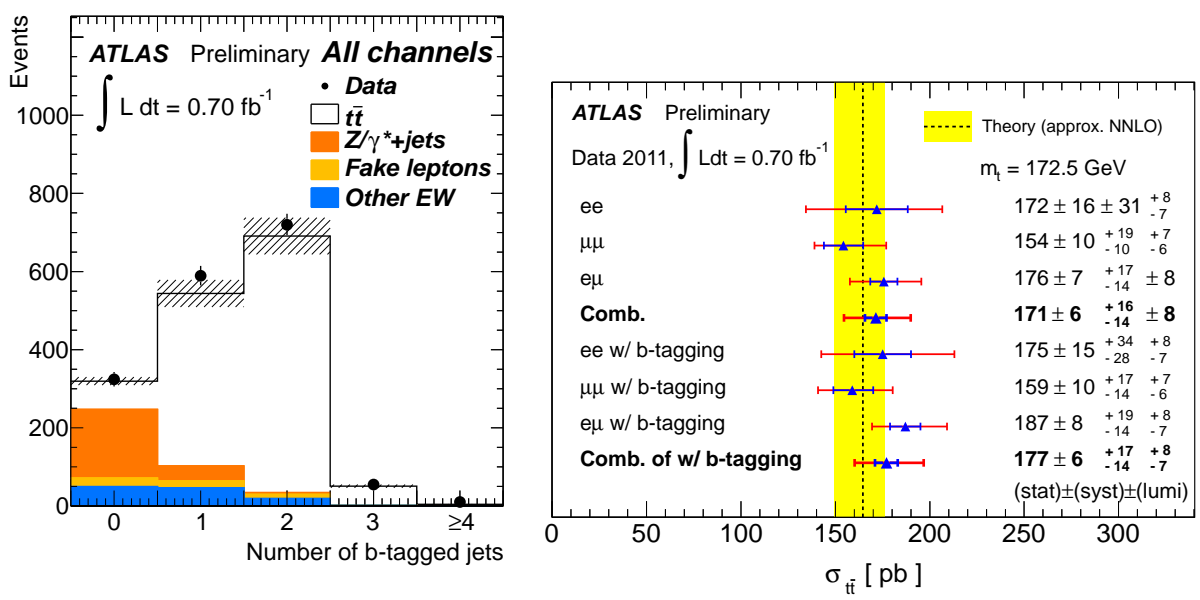

Figure 1: (Left) Multiplicity distribution of $b$-tagged jets in $e e, \mu \mu$ and $e \mu$ events. Contributions from diboson and single top-quark events are summarized as 'Other EW'. (Right) Cross-section summary in the dilepton channel.

$\mu \mu$ channels must satisfy $E_{T}^{\text {miss }}>60 \mathrm{GeV}$, to suppress multi-jet background, and $m_{\ell \ell}$ must differ by at least $10 \mathrm{GeV}$ from the $Z$-boson mass to suppress background from $Z / \gamma^{*}+$ jets. For the $e \mu$ channel, instead of cuts on $E_{T}^{\text {miss }}$ and $m_{\ell \ell}, H_{T}$, defined as the scalar sum of the transverse energies of the two selected leptons and all selected jets, must satisfy $H_{T}>130 \mathrm{GeV}$. For the analysis requiring one $b$-tagged jet, the $E_{T}^{\text {miss }}$ requirement for $e e$ and $\mu \mu$ channel was relaxed to $40 \mathrm{GeV}$, while the $H_{T}$ requirement for $e \mu$ events was set at $H_{T}>140 \mathrm{GeV}$.

The cross-section was extracted using a cut and count method, where the number of expected background events are subtracted from the number of candidate events passing the selection criteria, and the cross-section is measured taking into account the $t \bar{t}$ signal acceptance obtained from Monte Carlo. Background contributions from $Z / \gamma^{*}+$ jets and events with misidentified leptons are evaluated directly from data. The contribution for other electroweak background processes with two real leptons, such as single top quarks, $Z \rightarrow \tau \tau, W W, Z Z$ and $W Z$ production are determined from Monte Carlo simulations. In Figure 1 (left) the number of selected $b$-tagged jets and the expectation for $0.70 \mathrm{fb}^{-1}$ is shown for the analysis with $b$-tagging requirement after applying all selection criteria except the $b$-tagged jet multiplicity cut, with the three channels combined (ee, $\mu \mu$ and $e \mu$ ). For both analyses, the results of the three channels were combined using a likelihood fit. The impact of systematic uncertainties from leptons, jets, pile-up, theoretical cross-sections, data driven methods, generation and simulation were included into the likelihood fit.

Figure 1 (right) summarizes the cross-sections extracted for the individual channels and for the combination of all channels for the the two complementary analyses with and without $b$-tagging requirement. Dominant systematics come from the luminosity and jet energy scale uncertainties, being the $b$-tagging calibration uncertainty also an important source for the analysis using $b$-tagging. 


\section{Measurement of the $t \bar{t}$ production cross-section in the single lepton channel}

Single lepton $t \bar{t}$ events, , which corresponds to about $30 \%$ of the $t \bar{t}$ decays, are characterised by the presence of an electron or muon, jets and missing transverse energy in the final state. The events selected for analysis were triggered by a single lepton trigger. They were required to contain exactly one reconstructed lepton with $p_{T}>20 \mathrm{GeV}$, matching the corresponding high level trigger object. The $E_{T}^{\text {miss }}$ was required to be greater than 35 (20) $\mathrm{GeV}$ in the electron (muon) channel and the transverse leptonic $W$ mass was required to be greater than $25 \mathrm{GeV}\left(60 \mathrm{GeV}-E_{T}^{\text {miss }}\right)$ in the electron (muon) channel. The requirement was stronger in the electron channel to suppress the larger multijet background. Finally, events were required to have three or more jets with $p_{T}>25 \mathrm{GeV}$ and $|\eta|<2.5 \mathrm{GeV}$.

The main backgrounds to $t \bar{t}$ signal events arise from $W$-boson production in association with jets (in which $W$ decays leptonically) and from QCD multi-jet production. Relatively smaller backgrounds also arise from $Z+$ jets, diboson and single top production. The multi-jet background was measured with a data-driven approach. The other smaller backgrounds have been estimated from MC simulations, normalized to the latest theoretical predictions. The MC simulation is expected to predict correctly the $W+$ jet kinematical distributions, but not the relative normalizations of the different jet multiplicities, which are then extracted from the fit described below.

Two different approaches were used to extract the top quark pair cross-section from the full 35 $\mathrm{pb}^{-1}$ of data recorded in 2010. One exploits only the kinematic differences between $t \bar{t}$ and the dominant $W+$ jets background, while the other one adds $b$-tagging information to further separate signal and background. A likelihood discriminant is built from well modeled event kinematics variables, as uncorrelated as possible and leading to reduced statistical and jet energy scale uncertainties on the measured cross-section. The variables used are: aplanarity, lepton charge and pseudorapidity $\eta$ for the non- $b$-tag analysis; aplanarity, $H_{T, 3 p}$, as sum of the transverse momenta of all jets except for the leading two, normalized to the sum of longitudinal momenta of all reconstructed objects, lepton $\eta$ and average $b$-tagging algorithm output of the two most $b$-like jets for the $b$-tag analysis.

The $t \bar{t}$ production cross-section was extracted from a binned likelihood fit to data, in which the multi-jet and smaller backgrounds were fixed to their expected contributions. To provide maximum sensitivity, the fit is performed simultaneously to different subsamples: with 3 and $\geq 4$ jets (3, 4 and $\geq 5$ jets) for the non- $b$-tag ( $b$-tag) analyses in the electron and muon channels separately.

The analysis using $b$-tagging incorporates systematic uncertainties as nuisance parameters in the fit allowing data to constrain these uncertainties, while the uncertainties from the non- $b$-tag analysis are obtained from ensemble tests. The results of the fits are shown in Figure 2 (left).

The measured cross-section was $\sigma_{t \bar{t}}=186 \pm 10$ (stat. ${ }_{-20}^{+21}$ (syst.) \pm 6 (lumi.) for the analyses using $b$-tagging information and $\sigma_{t \bar{t}}=171 \pm 17$ (stat.) ${ }_{-17}^{+20}$ (syst.) \pm 6 (lumi.) for the one not relying on $b$-tagging. Systematic uncertainties are dominated by the $b$-tagging calibration and the knowledge of the heavy flavour fraction of the $W+$ jets background for the analysis using $b$-tagging and by the initial and final state radiation modeling and jet energy scale uncertainties for the analysis without $b$-tagging. 

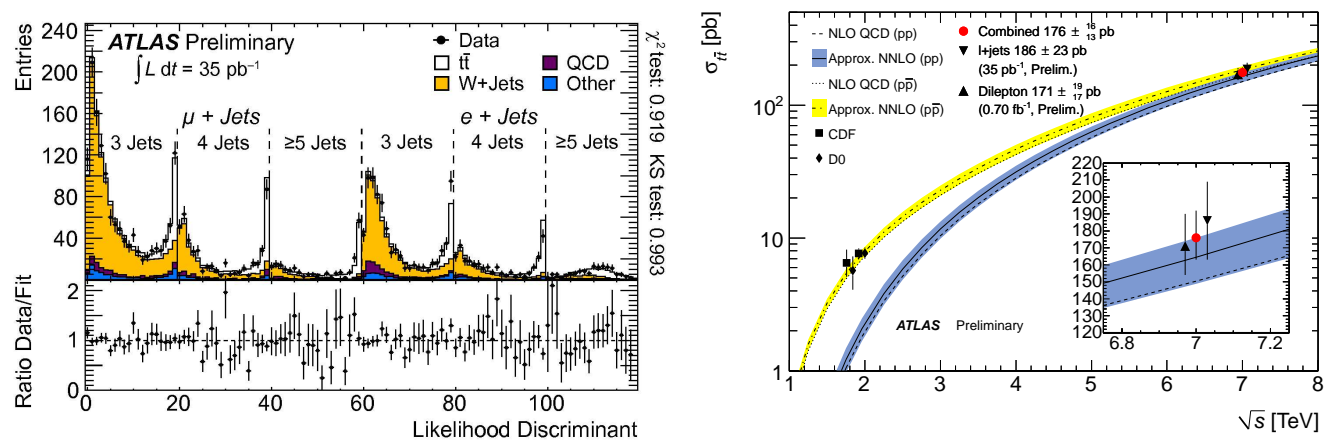

Figure 2: (Left) Distribution of the likelihood discriminant $\mathrm{D}$ for the single lepton analysis using $b$ tagging. (Right) Dependence of $\sigma_{t \bar{t}}$ on $\sqrt{s}$.

\section{Combination of $t \bar{t}$ cross-section measurements in lepton channels and conclusions}

A combination of the measurements obtained in the dilepton channel with $0.70 \mathrm{fb}^{-1}$ of data taken in 2011 without using $b$ tagging information and in the single lepton channel using $b$-tagging with the full $35 \mathrm{pb}^{-1}$ of 2010 data was performed. Common sources of systematic uncertainties in the different analyses were treated consistently in order to perform the combination. The result obtained was $\sigma_{t \bar{t}}=176 \pm 5$ (stat. $)_{-20}^{+13}$ (syst.) \pm 6 (lumi.). The dominant systematic uncertainties come from $W+$ jets heavy flavour content, the dilepton fake lepton background estimate, reconstructed jet identification efficiency, jet energy scale and initial and final state radiation.

Figure 2 (right) summarizes the measurements of the $t \bar{t}$ cross-section obtained from Tevatron and LHC experiments overlaid on the theoretical predictions as a function of the center-of-mass energy. The ATLAS combined measurement presented in this note, which has a total error of $8.2 \%$ is approaching the precision of recent Tevatron cross-section combinations. The precision achieved is significantly better than that obtained in the previous ATLAS measurement based on 2010 data [9]. The result shows a good agreement with the SM predictions as well as with the CMS measurement. In the all hadronic channel, a search for $t \bar{t}$ production was performed with $36 \mathrm{pb}^{-1}$ of data collected in 2010, setting a 95\% confidence level upper limit of $261 \mathrm{pb}$, compatible with the predicted SM cross-section and the ATLAS measurements. Measurements of the cross-section in this channel, as well as in the tau final states channels are in progress, together with differential cross-sections and measurements of the associated production of jets in $t \bar{t}$ events.

\section{References}

[1] S. Moch and P. Uwer, Phys. Rev. D 78 (2008) 034003.

[2] U. Langenfeld, S. Moch, and P. Uwer, Proc. XVII Int. Workshop on Deep-Inelastic Scattering and Related Topics, dx.doi.org/10.3360/dis.2009.131, arXiv:hep-ph/0907.2527.

[3] M. Beneke et al., Phys. Lett. B 690 (2010) 483;

[4] T. Affolder et al., CDF Collaboration, Phys. Rev. D 64 (2001) 032002, erratum-ibid. D 67 (2003) 119901. 
[5] T. Aaltonen et al., CDF Collaboration, Phys. Rev. Let. 105 (2010) 012001.

[6] V. M. Abazov et al., D0 Collaboration, Phys. Rev. D 67 (2003) 012004.

[7] V. M. Abazov et al., D0 Collaboration, to appear in Phys. Rev. D, arXiV:hep-ex/1101.0124.

[8] The CMS Collaboration, Phys. Lett. B 695 (2011) 424.

[9] The ATLAS Collaboration, Eur. Phys J. C 71 (2011) 1577.

[10] The ATLAS Collaboration, ATLAS-CONF-2011-100.

[11] The ATLAS Collaboration, ATLAS-CONF-2011-035.

[12] The ATLAS Collaboration, ATLAS-CONF-2011-023.

[13] The ATLAS Collaboration, ATLAS-CONF-2011-066.

[14] The ATLAS Collaboration, ATLAS-CONF-2011-108. 